Check for updates

Cite this: RSC Adv., 2018, 8, 39463

Received 18th August 2018

Accepted 7th November 2018

DOI: $10.1039 / \mathrm{c} 8 \mathrm{ra06922k}$

rsc.li/rsc-advances

\section{High-water-absorbing calcium alginate fibrous scaffold fabricated by microfluidic spinning for use in chronic wound dressings $\uparrow$}

\author{
Jie Cai, ${ }^{a}$ Xiaojing Chen, ${ }^{\text {bc }}$ Xiaojing Wang, ${ }^{d}$ Yulu Tan, ${ }^{a}$ Dongdong Ye, ${ }^{a}$ Yongtang $\mathrm{Jia}{ }^{\mathrm{a}}$ \\ Peifeng Liu ${ }^{b c}$ and Hui Yu (D)*a
}

\begin{abstract}
More and more water-absorbing wound dressings have been studied since moist wound-healing treatment can effectively promote the healing of wounds. In this work, we introduce a novel method to produce improved wound dressings with high-water-absorbance. A high-water-absorbing calcium alginate (CaAlg) fibrous scaffold was fabricated simply by microfluidic spinning and centrifugal reprocessing. The structure and physical properties of the scaffold were characterized, and its water-absorbing, cytotoxicity properties and other applicability to wound dressings were comprehensively evaluated. Our results indicate that this material possesses high water-absorbing properties, is biocompatible, and has a 3D structure that mimics the extracellular matrix, while Ca-Alg fibers loaded with silver nanoparticles (AgNPs) exhibit broad-spectrum antibacterial activities; these properties meet the requirements for promoting the healing of chronic wounds and are widely applicable to wound dressings.
\end{abstract}

\section{Introduction}

A wound is an injury to normal tissue caused by external physical, chemical, biological, or other types of damage. Based on healing time, wounds are categorized as acute wounds (such as those resulting from surgical incisions and mechanical injuries) and chronic wounds (such as decubitus ulcers and diabetic foot ulcers). The increasing prevalence of chronic wounds, such as decubitus ulcers and ulceration, is closely associated with the elderly and the aging population. These types of chronic wound take long times to heal and are often accompanied by extensive wound exudates that can easily cause wound infection and ulceration if not promptly removed, thereby further complicating the wound-healing process. If an infection spreads, it can lead to life-threatening complications, such as sepsis, among others. ${ }^{1,2}$ A series of new functional wound dressings has been developed in recent years for accelerating the healing of these types of chronic wound.

${ }^{a}$ The Engineering Technology Research Center for Functional Textiles in Higher Education of Guangdong Province, College of Textile Materials and Engineering, Wuyi University, Jiangmen 529020, China. E-mail: yuhuihui_2000@163.com; Tel: +86750 3296233

${ }^{b}$ State Key Laboratory of Oncogenes and Related Genes, Shanghai Cancer Institute, Renji Hospital, School of Medicine, Shanghai Jiao Tong University, Shanghai 200032, China

${ }^{c}$ Central Laboratory, Renji Hospital, School of Medicine, Shanghai Jiao Tong University, Shanghai 200127, China

dJiangmen Central Hospital, Jiangmen, Guangdong 529030, China

$\dagger$ Electronic supplementary information (ESI) available. See DOI: $10.1039 / \mathrm{c} 8 \mathrm{ra06922k}$
In 1962, based on animal experiments, Winter introduced "moist wound-healing theory", which suggested that a moist environment doubles the rate of wound healing compared to a dry environment, as a moist environment facilitates growthfactor release and cell proliferation that accelerates the migration of basal epidermal cells into the wound. ${ }^{3} \mathrm{~A}$ moist environment can effectively promote the healing of chronic wounds, such as decubitus ulcers and ulceration, provided that the wound is clean. On the basis of this theory, modern wound dressings with water absorption and moisture-retention features were fabricated in the forms of films, foams, hydrocolloids, hydrogels, and fibers. Among them, fiber wound dressings are well ventilated and possess greater surface areas and controllable structures, compared to other types of dressing. ${ }^{4-7}$ Hence, fiber wound dressings have become the focus of new wound-dressing research.

Alginate is a natural, non-toxic, hemostatic, biodegradable, and biocompatible linear polysaccharide extracted from brown algae. It has been widely used in wound dressings with wellrecognized clinical therapeutic efficacy. It is an ideal wound filler that is softer, easier to fold, and easier to apply compared to other wound-dressing materials. ${ }^{8-11}$ In addition, alginatebased wound dressings are highly water absorbent compared to conventional dressings; they are capable of absorbing 15-17 times their own weight in physiological saline solution, equivalent to 5-7 times that of traditional gauze. ${ }^{12-16}$ Currently, most alginate-based wound dressings are fabricated from Ca-Alg. These wound dressings can absorb an extensive amount of wound exudate and expand upon contact with the wound. Ionexchange reactions between calcium ions in the dressing and 
sodium ions in the wound exudate results in the formation of a hydrogel layer on the wound surface, providing a moist environment that promotes wound healing. ${ }^{17,18}$

Microfluidic techniques have been widely used in recent years for biological detection, drug screening, substance isolation, gene sequencing, and in vitro diagnosis, among others, due to advantages that include low loading volumes, rapid detection, cost effectiveness, and high portability. ${ }^{19-22}$ Many research groups (such as S. H. Lee, J. Qin, Q. Liang, et al.) have proposed and used microfluidic spinning to fabricate a series of fibers with varying morphologies, which broadens the applications of microfluidic chips. ${ }^{23-34}$ For instance, Jun et al. (2014) encapsulated cells in fibers by microfluidic spinning to fabricate a bottom-up biomedical porous material for tissueengineering studies. ${ }^{24-29}$ In addition, Yu et al. (2014) fabricated an encodable biomimetic bamboo-like hybrid microfiber, while Xie et al. (2018) fabricated necklace-like microfibers using microfluidic spinning and developed other tissue-engineering applications for these fibers by integrating them with various types of cells and growth factors. ${ }^{30-34}$

Most pure natural polymers suitable for wound dressings can be prepared by microfluidic spinning in an aqueous environment devoid of organic solvents. On the other hand, the basic materials for electrospinning are polymers and solvents, commonly organic solvents, which are disadvantageous because some of them are toxic or highly cytotoxic. Consequently, there is a significant hurdle for the use of electrospun fibers in biomedical-engineering and wound-dressing applications. In contrast to electrospinning, microfluidic spinning, in which the materials are usually cell-friendly, is more suitable for wound-dressing applications. In addition, microfluidic spinning uses an eco-friendly aqueous system, which is more environmental friendly than electrospinning, and the electrospinning of pure natural polymers is generally difficult. ${ }^{23}$ To the best of our knowledge, to date, alginate, a common polysaccharide, has not been electrospun on its own, due to its high viscosity and intermolecular repulsions between its anionic groups. ${ }^{35}$ However, Ca-Alg fibers are easily fabricated by microfluidic spinning using pure aqueous sodium alginate solutions; organic solvents are not used throughout the entire microfluidic-spinning process, which is not possible using other spinning methods. For instance, electrospinning uses electrostatic forces and has strict voltage, humidity, and temperature requirements. On the other hand, microfluidic spinning is based on micro-scale fluid dynamics. The factors that influence microfluidic spinning are different to those of the bulk fluid due to surface-tension and energy-dissipation differences, as well as fluidic resistance. ${ }^{23}$ In addition, the preparation conditions for microfluidic spinning have no special voltage, humidity, or temperature requirements. The microfluidic-spinning process also involves a mild and wet environment, which contributes to the encapsulation of sensitive materials such as cells. Above all, the above-mentioned characteristics provide certainty when preparing safe, nontoxic and bioactive wound dressings.

The aforementioned features of moist wound-healing theory and microfluidic spinning inspired us to study new wound dressings that are applicable to the treatment of chronic wounds with extensive exudates, such as decubitus ulcers and ulcerations. In this study, microfluidic spinning was used to fabricate a calcium alginate (Ca-Alg) fibrous scaffold for wounddressing use. There are following novelty points in our work. Firstly, there was rare to study the application of fibrous scaffold prepared via microfluidic spinning technology for wound healing; secondly, Ca-Alg fibrous scaffold not only possessed excellent biocompatibility and degradability, but also could fill the wound since its 3D structure, as the degradation speed of Ca-Alg fibrous scaffold was very fast, and its biocompatibility was outstanding, therefore, it can fused together with the new tissue without changing the wound dressings, which can accelerate the wound healing; thirdly, Ca-Alg fibrous scaffold had high porosity $(94.4 \%)$ that could better to facilitate gas exchange between the wound and the outside environment, the high contact area of material and wound (specific surface area: $14.8 \mathrm{~m}^{2} \mathrm{~g}^{-1}$ ), which were beneficial to accelerate the healing of wound; in addition, the Ca-Alg fibrous scaffold possessed the higher water absorbing ( $35 \mathrm{~g} \mathrm{~g}^{-1}$ ) than the traditional calcium alginate fiber (7-15 $\left.\mathrm{g} \mathrm{g}^{-1}\right) \cdot{ }^{\mathbf{1 2 - 1 6}}$ Its structural and morphological features were characterized using biological microscopy, scanning electron microscopy (SEM), inductively coupled plasma mass spectrometry (ICP-MS), mercury-intrusion porosimetry (MIP), and X-ray diffraction (XRD).

\section{Experimental}

\subsection{Materials}

Sodium alginate (Na-Alg) (viscosity $=1.05-1.15$ Pa $\mathrm{s}$ ) was purchased from Tianjin Fuchen Chemistry Reagent Factory (Tianjin, China). Calcium chloride (anhydrous) $\left(\mathrm{CaCl}_{2}\right)$ (A.R.) was obtained from Xilong Chemical Co., Ltd. (Guangzhou, China). AgNP solution (0.1 $\left.\mathrm{mg} \mathrm{mL}^{-1}, 20 \mathrm{~nm}\right)$ was purchased from Nanjing XFNANO Materials Tech Co., Ltd. (Nanjing, China). L929 mouse fibroblasts were obtained from the Shanghai Cancer Institute (Shanghai, China). Dulbecco's modified Eagle's medium (DMEM) and Cell Counting Kit-8 (CCK8) reagent were purchased from Paisley (UK). E. coli and $S$. aureus were obtained from Shanghai Luwei Technology Co., Ltd. (Shanghai, China). Nutrient agar plates were purchased from Shanghai Shenqi Biotechnology Co., Ltd. (Shanghai, China). Customized microfluidic chips used in this work were tailor made by the Dalian Institute of Chemical Physics, Chinese Academy of Sciences (Dalian, China).

\subsection{Fabrication of the calcium alginate fibrous scaffold}

A $\mathrm{CaCl}_{2}$ coagulation solution ( $2 \mathrm{wt} \%$ ) and a Na-Alg spinning solution ( $2 \mathrm{wt} \%, 421 \mathrm{mPa}$ s) were loaded into separate $2 \mathrm{~L}$ reagent bottles connected to a fluid-injection system (homemade) and a $10 \mathrm{~mL}$ syringe was fixed on the LSP02-1B microinjection pump (Longer Precision Pump Co., Ltd.), respectively. Fig. 1(a) shows that both solutions were respectively fed into channels A and B of the microfluidic chip, which was positioned in a Petri dish (diameter $=12 \mathrm{~cm}$ ). Subsequently, $80 \mathrm{~mL}$ of the 2 wt $\% \mathrm{CaCl}_{2}$ solution was added to the Petri dish. The solutions 


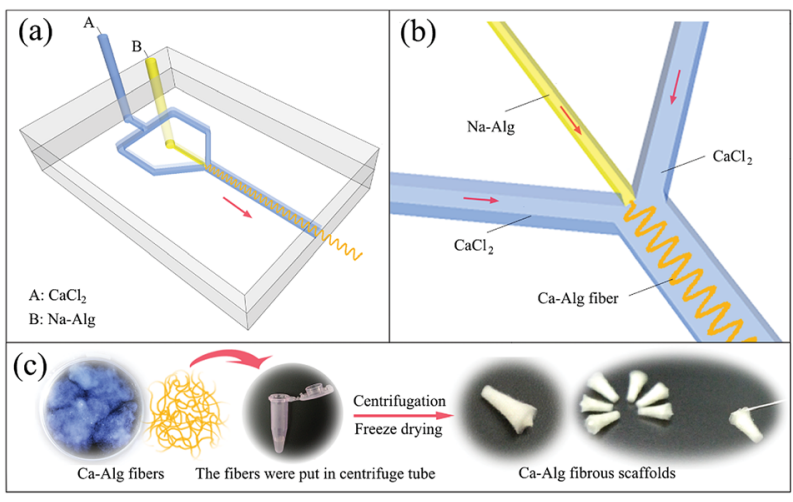

Fig. 1 The schematic diagram was the process of fabrication Ca-Alg fibrous scaffolds using microfluidic spinning technology. (a) The structure diagram of microfluidic chip; (b) enlarged view of crossing section of A and B fluids; (c) the preparation process of Ca-Alg fibrous scaffolds.

were continuously injected at $200 \mathrm{~mL} \mathrm{~h}^{-1}$ for $\mathrm{CaCl}_{2}$ and $0.5 \mathrm{~mL}$ $\mathrm{h}^{-1}$ for Na-Alg. As shown in Fig. 1(b), calcium alginate (Ca-Alg) fiber formed due to rapid $\mathrm{Na}^{+} / \mathrm{Ca}^{2+}$ exchange at the intersection within the chip.

The process used to fabricate the Ca-Alg fibrous scaffold is shown in Fig. 1(c). Briefly, Ca-Alg fibers were positioned in $1.5 \mathrm{~mL}$ centrifuge tubes and placed in a centrifuge (TGL-16B, Shanghai Anting Scientific Instrument Factory), and centrifuged at $10000 \mathrm{rpm}(10 \mathrm{k} \mathrm{rpm})$ for $10 \mathrm{~min}$. After centrifugation, the resulting supernatant was discarded and the centrifuge tubes were frozen at $-16{ }^{\circ} \mathrm{C}$ for $24 \mathrm{~h}$, followed by vacuum lyophilization in a freeze dryer (Lab-1-80, Beijing Boyikang Laboratory Apparatus Co., Ltd.) for $24 \mathrm{~h}$.

Ca-Alg fibers loaded with AgNPs were prepared as described for the Ca-Alg fibers. The $2 \mathrm{wt} \% \mathrm{Na}-\mathrm{Alg}$ and $0.1 \mathrm{mg} \mathrm{mL}^{-1} \mathrm{AgNP}$ solutions were evenly mixed at a $2: 1$ mass ratio to prepare a spinning solution of Na-Alg loaded with AgNPs.

\subsection{Characterization}

2.3.1. Biologic microscope and SEM. One Ca-Alg fiber and its water-absorbed scaffold were separately placed on glass slides and excess water was removed with filter paper. Morphologies were examined by biological microscopy (OLYMPUS-CX31, Olympus Co., Japan).

An appropriate amount of the fibers and the fibrous scaffold were fixed onto electron-microscope stages with conductive resins, after which they were coated with gold for $30 \mathrm{~s}$. The morphologies of the gold-coated materials were examined by scanning electron microscopy (SEM, SU8010, Hitachi, Japan) at a $5 \mathrm{kV}$ accelerator voltage.

2.3.2. MIP. The pore structure of the scaffold was characterized by mercury-intrusion porosimetry (MIP, PoreMaster-60, Quantachrome Instruments Co., Ltd., USA) in mercuryintrusion-scanning mode under the following conditions: the sample was dried at $40{ }^{\circ} \mathrm{C}$ for $48 \mathrm{~h}$ and examined at a mercurysolid contact angle of $140^{\circ}$ and a mercury surface tension of $0.48 \mathrm{~N} \mathrm{~m}^{-1}$.
2.3.3. XRD. XRD was performed using an X-ray diffractometer (Bruker D8, Bruker Corporation, Germany) equipped with a copper target as the diffraction source, and at a voltage of $40 \mathrm{kV}$, a current of $300 \mathrm{~mA}$, a scanning speed of $5^{\circ} \mathrm{min}^{-1}$, and a $10-90^{\circ}$ scanning range.

2.3.4. ICP-MS. The silver content of the Ca-Alg fibers loaded with AgNPs were measured by inductively coupled plasma optical emission spectrometry (ICP-OES, Agilent Technologies, Inc., USA) under the following conditions: $1 \mathrm{~kW}$ of power, a 15 $\mathrm{L} \min ^{-1}$ plasma gas flow rate, a $1.5 \mathrm{~L} \mathrm{~min}^{-1}$ auxiliary gas flow rate, a $0.75 \mathrm{~L} \mathrm{~min}^{-1}$ nebulizer gas flow rate, and a $15 \mathrm{rpm}$ pump speed.

\subsection{Water absorption measurements}

The Ca-Alg fibrous scaffold was weighed (recorded as $m_{0}$ ) and added to $20 \mathrm{~mL}$ of distilled water in a $5 \mathrm{~cm}$ Petri dish. After immersion in water for $1 \mathrm{~h}$ at room temperature, the scaffold was removed using forceps and weighed (recorded as $m_{1}$ ) after being blotted with a filter paper to remove excess water from the surface. The water absorption coefficient $(Q)$ was calculated using the following equation:

$$
Q=\frac{m_{1}-m_{0}}{m_{0}} \times 100 \%
$$

The water absorption of the Ca-Alg scaffold was compared with that of a Ca-Alg 2D membrane, which was fabricated and examined in parallel. Briefly, a $2 \mathrm{~cm}$ rubber ring was first placed in a Petri dish that was filled with $2 \mathrm{wt} \% \mathrm{CaCl}_{2}$ solution, after which $2 \mathrm{wt} \%$ sodium alginate solution was poured over the center of the rubber ring until the ring was entirely covered. Rapid ion exchange between the two solutions led to the formation of a Ca-Alg 2D membrane, which was then cooled to $-16{ }^{\circ} \mathrm{C}$ for $24 \mathrm{~h}$ and further subjected to vacuum lyophilization for $24 \mathrm{~h}$ in a freeze dryer. The Ca-Alg $2 \mathrm{D}$ membrane was subjected to the same water-absorption testing as described for the above-mentioned Ca-Alg fibrous scaffold $(n=3)$.

\subsection{Structural stability testing}

Ca-Alg fibers were loaded in $1.5 \mathrm{~mL}$ centrifuge tubes and separately centrifuged at $4 \mathrm{k}, 6 \mathrm{k}, 8 \mathrm{k}$, and $10 \mathrm{k} \mathrm{rpm}$ for $10 \mathrm{~min}$. Excess water in the upper part of the centrifuge tube was then removed by pipette. The Ca-Alg fibrous scaffolds were prepared following freeze drying. Each prepared sample was then placed in a $5 \mathrm{~cm}$-diameter Petri dish, $20 \mathrm{~mL}$ of distilled water was added and the morphology was examined after incubation for $1 \mathrm{~h}$ at room temperature.

\subsection{Cell-proliferation assay}

Cell-proliferation rates in the presence of $1 \mathrm{mg} \mathrm{mL}^{-1}$ of the CaAlg fibrous scaffold was assessed in CCK8 assays using physiological saline solution as the blank control group. An L929 cell suspension in DMEM containing $10 \%$ fetal bovine serum was seeded into a 96-well plate at $8 \times 10^{3}$ cells per well and grown to the logarithmic phase at $37{ }^{\circ} \mathrm{C}$ and $5 \% \mathrm{CO}_{2}$, after which experimental samples were added and incubation continued for 
a further $24 \mathrm{~h}$. The medium was then discarded and replaced with fresh serum- and antibiotic-free DMEM containing 10\% CCK8 reagent, after each well was rinsed twice with physiological saline solution. The plate was further incubated at $37{ }^{\circ} \mathrm{C}$ and $5 \% \mathrm{CO}_{2}$ for $1 \mathrm{~h}$. Each sample was tested five times. The growth status of the cells was determined using a $\mathrm{ZOE}^{\mathrm{TM}}$ inverted microscope (Bio-Rad Laboratories, Inc., USA), and the $\mathrm{OD}_{450}$ value for each well was measured using a SYNERGYTM Multi-Mode microplate reader (Biotek Instruments, Inc., USA) in order to calculate the relative growth rate (RGR\%), as follows: $\mathrm{RGR} \%=\mathrm{ODt} / \mathrm{ODnc} \times 100 \%$, where ODt and ODnc refer to the absorbance values of the sample and blank groups, respectively.

\subsection{Antibacterial properties}

Both Ca-Alg fibers and Ca-Alg fibers loaded with AgNP materials were subjected to antibacterial assays using the bacteriostatic ring method. Prior to each experiment, samples loaded on $8 \mathrm{~cm}$ discs were sterilized by ultraviolet radiation for 2 h. E. coli and $S$. aureus $(0.3 \mathrm{~mL})$ broth cultures were aliquoted and spread onto the surfaces of nutrient agar plates using a coated rod. The surfaces of the agar plates were allowed to dry for $15 \mathrm{~min}$ before sample discs of Ca-Alg fibers and Ca-Alg fibers loaded with AgNPs were placed onto the plates. The agar plates were then incubated in an MJX Intelligent Incubator (Ningbo Jiangnan Instrument Factory) at $37^{\circ} \mathrm{C}$ for $16 \mathrm{~h}$. The samples on the agar plates were then examined, and the diameters of the inhibition zones were measured.

\subsection{Statistical analyses}

The experimental data were analyzed using SPSS13.0 statistical software. A between-group comparison was performed by oneway analysis of variance, and $\alpha=0.05$ was selected as the threshold for statistical significance.

\section{Results and discussion}

\subsection{Physico-morphological structures}

The physico-morphological structures of Ca-Alg fibers and the Ca-Alg fibrous scaffold were examined by biological microscopy and SEM. The diameter of Ca-Alg fibers would increase as the velocity of the Na-Alg solution increase (Fig. S1 and S2†), on the contrary, the diameter would reduce as the velocity of the $\mathrm{CaCl}_{2}$ solution increase (Fig. S3 and S4 $\dagger$ ). The diameter of the dry fibers increased 1.4 times after thoroughly swollen (Fig. S5†). As

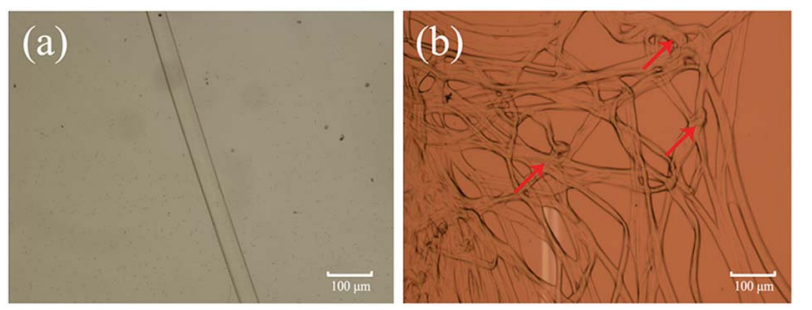

Fig. 2 Biologic microscope images of Ca-Alg fibers and Ca-Alg fibrous scaffold in water absorption state. (a) Ca-Alg fiber $\times 10$; (b) $\mathrm{Ca}$ Alg fibrous scaffold $\times 10$. shown in Fig. 2(a), the Ca-Alg fibers exhibit continuous and uniform fibrous morphologies with smooth surfaces following absorption of water. Fig. 2(b) reveals that the Ca-Alg fibrous scaffold has a good uniform porous structure with some fusion points at the fibers junctions, as indicated by arrows, which are ascribable to the intertwisting and merging of soft Ca-Alg fibers under high centrifugal force; as a result, the structure of the scaffold was formed through physical bonding.

Fig. 3 displays SEM images of the Ca-Alg fibers and the CaAlg fibrous scaffold, which reveals that Ca-Alg fibers have porous structures with uniform diameters, as shown (Fig. 3(a and b)). Following centrifugation, the Ca-Alg fibers were densely intertwined and some fibers were broken (Fig. 3(c)); bonding points at fiber intersections are clearly observable in Fig. 4(d), as indicating by arrows. The above results indicate that at an effective centrifugal force, the bonding forces between the fibers in the scaffold are sufficiently large to ensure the structural stability of the scaffold.

MIP measurements reveal that the Ca-Alg fibrous scaffold has a porosity of $94.4 \%$ and an average pore diameter of $3.9 \mu \mathrm{m}$ (Fig. 4). The densely interconnected porous framework renders the Ca-Alg fibrous scaffold well ventilated and facilitates the metabolic exchange of nutrients and water between the wound and the external environment. The scaffold can also absorb a large amount of wound exudate in order to create a moist environment, thereby favoring wound healing. ${ }^{36-38}$ As shown in Fig. S6, $\uparrow$ the compression resistance of the Ca-Alg fibrous scaffold was about $5 \mathrm{MPa}$, indicating excellent mechanical properties as a kind of wound dressings.

\subsection{Chemical characterization}

In this study, we investigated the chemical properties of Ca-Alg fibers and the Ca-Alg fibers loaded with AgNPs by XRD and ICPMS. The XRD spectra shown in Fig. 5 reveal a pronounced amorphous peak at $2 \theta=21.4^{\circ}$ for the Ca-Alg fibers (Fig. 5(a)); however, following the addition of AgNPs, a crystal diffraction peak corresponding to microcrystalline AgNPs (111) was

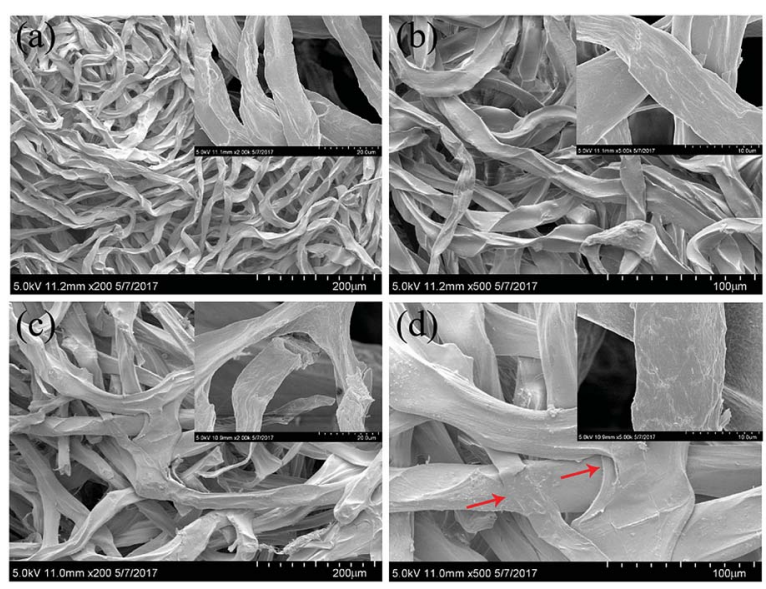

Fig. 3 The SEM images of Ca-Alg fibers and the Ca-Alg fibrous scaffold. (a) Ca-Alg fibers $\times 200$; (b) Ca-Alg fibers $\times 500$; (c) Ca-Alg fibrous scaffold $\times 200$; (d) Ca-Alg fibrous scaffold $\times 500$. 


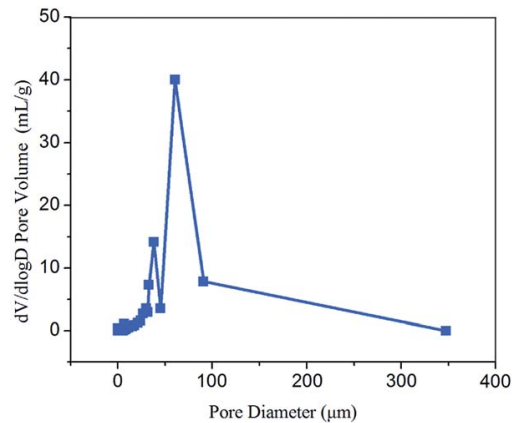

Fig. 4 Pore size distribution of Ca-Alg fibrous scaffold.

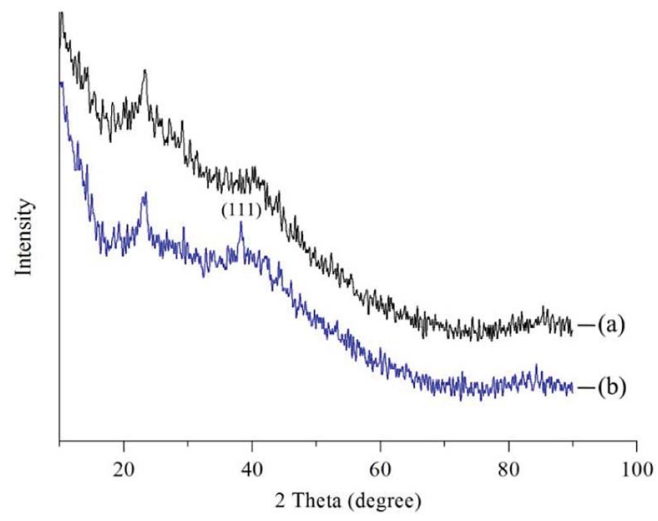

Fig. 5 XRD spectra of (a) Ca-Alg fibers and (b) Ca-Alg fibers loaded with AgNPs.

observed at $2 \theta=38.1^{\circ}$, in addition to the amorphous peak for Ca-Alg (Fig. 5(b)), which confirms the presence of AgNPs in the Ca-Alg fibers loaded with AgNPs. The ICP-OES spectrum revealed a $\mathrm{Ag}$ content of $4030.9 \mathrm{mg} \mathrm{kg}{ }^{-1}$, which also supports the existence of elemental $\mathrm{Ag}$ in the Ca-Alg fibers loaded with the AgNP-containing scaffold.

\subsection{Water absorbability testing}

As shown in Fig. 6(a), the water absorbing capacity of the Ca-Alg fibrous scaffold was compared with that of the two-dimensional

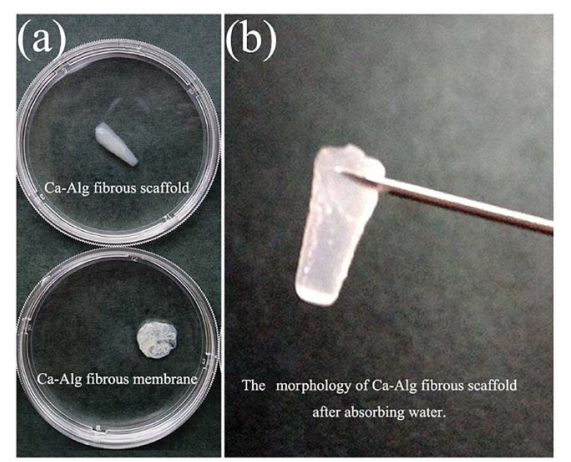

Fig. 6 Water absorption test of Ca-Alg fibrous scaffold (a) the experimental image of water absorption test for Ca-Alg fibrous scaffold and 2D membrane; (b) the morphology of the Ca-Alg fibrous scaffold after water absorption test.
(2D) Ca-Alg membrane, which served as the control. Fig. 6(b) reveals that the scaffold retains its original morphology following water absorption. Calculations show that the water absorbing capacity of the Ca-Alg fibrous scaffold (3573\%) was 3.9 times that of the Ca-Alg 2D membrane (908\%). In addition to the excellent water absorbing ability of the Ca-Alg itself, the Ca-Alg fibrous scaffold contains densely distributed micronsized pores that effectively improve water absorption by facilitating the retention of larger amounts of water. ${ }^{25,39}$

In this study, the Ca-Alg fibrous scaffold fabricated by microfluidic spinning was shown to absorb 35.7 times its own weight in water; this property can be used in wound dressings to absorb extensive wound exudate. In addition, this material can form a soft and adhesive hydrogel layer that seals the wound, ensuring a moist environment that is conducive to wound healing. Hence, the Ca-Alg fibrous scaffold is suitable for wound-healing applications.

\subsection{Structural stability testing}

Ca-Alg fibrous scaffolds were fabricated at different centrifugal velocities $(4 \mathrm{k}, 6 \mathrm{k}, 8 \mathrm{k}$, and $10 \mathrm{k} \mathrm{rpm})$, the results of which are shown in Fig. 7(a). After absorbing water for $1 \mathrm{~h}$, the scaffolds swelled without changing their original shapes (Fig. 7(b)). When picked up by tweezers, the Ca-Alg fibrous scaffolds fabricated at low centrifugal velocities lost their original shapes, while that fabricated at a high centrifugal velocity (10k rpm) maintained its shape (Fig. 7(c)), which is ascribable to a denser intertwinement of Ca-Alg fibers induced by the high centrifugal force, which enhances the structural stability of the Ca-Alg fibrous scaffold. The Ca-Alg fibrous scaffold possesses excellent biodegradable in vitro. The compared experiment results of SEM images were shown in the Fig. S6, $\dagger$ the Ca-Alg fibrous scaffold (Fig. $S 7(\mathrm{a}-\mathrm{d}) \dagger$ ) exhibited poor permanence of retention characteristic compared with commercial products (Fig. S7(e1) $\dagger$ ). The degradation speed of our sample was the fastest in all test samples.

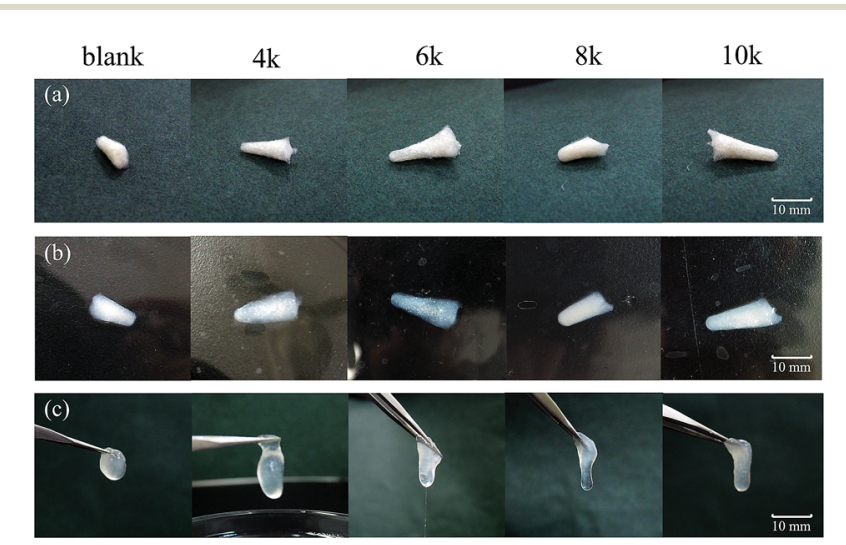

Fig. 7 The structural stability test. (a) The Ca-Alg fibrous scaffolds were prepared at different centrifugal velocity $(4 \mathrm{k}, 6 \mathrm{k}, 8 \mathrm{k}$ and $10 \mathrm{k} \mathrm{rpm})$; (b) the experimental images of the materials after absorbing water for $1 \mathrm{~h}$; (c) the experimental images of the materials when picked up by a tweeze. 


\subsection{Cytotoxicity}

Good biocompatibility is a prerequisite for materials used for treating wounds. Cytotoxicity assays are important in vitro biocompatibility assays for determining the biological responses of tissue following contact with a medical material. In this study, L929 mouse fibroblasts were studied in cell-proliferation assays to assess the cytotoxicity of the Ca-Alg fibrous scaffold. Fig. 8 reveals that, compared to the control group, adherent L929 cells displayed excellent proliferation and morphologies, with spherical as well as long spindle shapes in the presence of both Ca-Alg fibers and Ca-Alg fibers loaded with AgNPs. The cell-proliferation rates after $24 \mathrm{~h}$ of cultivation were $99.7 \pm 3.6 \%$ (Ca-Alg fibers) and $100.6 \pm 3.3 \%$ (Ca-Alg fibers loaded with AgNPs), with no significant differences compared to the control group ( $p>0.05)$, indicating that both Ca-Alg fibers and Ca-Alg fibers loaded with AgNPs are non-toxic, safe, and reliable materials that do not significantly affect L929 cell proliferation. Our results provide an experimental basis for further studies into the applications of this material in wound-healing treatments.

\subsection{Antibacterial properties}

One prerequisite for chronic wound dressings is that they show antibacterial properties that prevent infection of the wound. ${ }^{\mathbf{4 0}}$ In this study, we assessed the antibacterial properties of Ca-Alg fibers loaded with AgNPs and the control group (Ca-Alg fibers) against $E$. coli and $S$. aureus using the bacteriostatic ring method, the results of which are shown in Fig. 9. Compared to the control group, the Ca-Alg fibers loaded with AgNPs exhibited significant zones of inhibition, with diameters of $4.9 \mathrm{~mm}$ and $5.2 \mathrm{~mm}$, which indicates significant antibacterial activities against $E$. coli and $S$. aureus, respectively. On the other hand, the Ca-Alg fibers showed no bacteriostasis rings. The silver content increased with time point (Fig. S8†). That indicated that the antibacterial property is functional through the release of silver ions. These results indicate that the Ca-Alg fibers loaded with AgNPs and fabricated by microfluidic spinning release silver ions that are significantly antibacterial against both Gramnegative and Gram-positive bacteria. Hence, this material is useful as an antibacterial dressing for wound-healing applications.

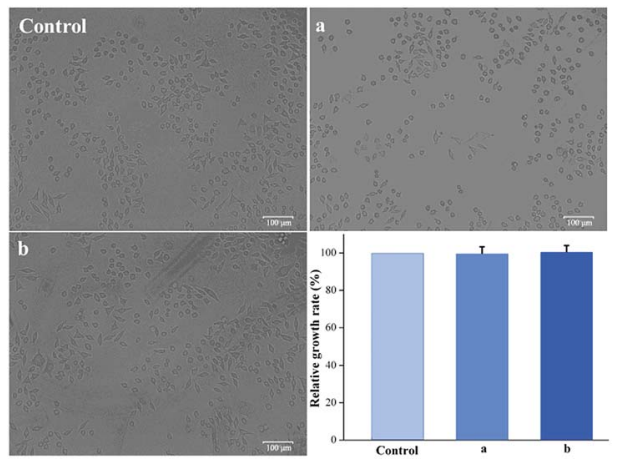

Fig. 8 The images of cell morphologies and cell-proliferation data (control: control group; (a) Ca-Alg fibers; (b) Ca-Alg fibers loaded with AgNPs).
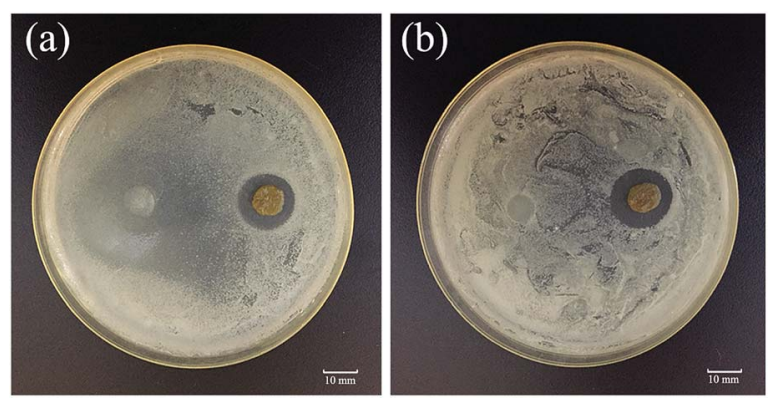

Fig. 9 The results of antibacterial assays using the bacteriostatic ring method for Ca-Alg fibers (left) and Ca-Alg fibers loaded with AgNPs (right). (a) E. coli; (b) S. aureus.

\section{Conclusions}

The Ca-Alg fibrous scaffold fabricated by microfluidic spinning is $94.4 \%$ porous and has an interconnected porous framework with a specific surface area of $14.8 \mathrm{~m}^{2} \mathrm{~g}^{-1}$. These pores facilitate the retention of large amounts of water and render the scaffold capable of absorbing 35.7 times its own weight in water, which creates a moist environment for wound healing. The cytotoxicity assay indicated that the biocompatibilities of Ca-Alg fibers and Ca-Alg fibers loaded with AgNPs are excellent, while the antibacterial assays indicate that the Ca-Alg fibers loaded with AgNPs exhibit significant antibacterial activities against $E$. coli and $S$. aureus. In summary, the Ca-Alg fibrous scaffold fabricated by microfluidic spinning can be used as a new type of wound dressing for the care of chronic wounds.

\section{Conflicts of interest}

There are no conflicts of interest to declare.

\section{Acknowledgements}

This work was supported by the National Natural Science Foundation of China (No. 81472842, 81502560), the Foundation of Higher Education of Guangdong, China (No. 2016KTSCX146) and Natural Science Foundation of Guangdong Province, China (No. 2018A0303130245).

\section{Notes and references}

1 M. P. Rowan, L. C. Cancio, E. A. Elster, D. M. Burmeister, L. F. Rose, S. Natesan, et al., Crit. Care, 2015, 19(1), 243-254.

2 K. A. Rieger, J. Mater. Chem. B, 2013, 1(36), 4531-4541.

3 G. D. Winter, Nature, 1962, 193(4812), 293-294.

4 C. Lagarde, P. J. M. Mulkens, R. Wimheuer and T. Bieber, J. Eur. Acad. Dermatol. Venereol., 1998, 11, S141.

5 C. T. Chiu, J. S. Lee, C. S. Chu, Y. P. Chang and Y. J. Wang, Mater. Med., 2008, 19(6), 2503-2513.

6 Y. Suzuki, Y. Nishimura, M. Tanihara, K. Suzuki, A. K. Kitahara, Y. Yamawaki, et al., J. Artif. Organs, 1998, 1(1), 28-32. 
7 F. A. Paskiabi, S. Bonakdar, M. A. Shokrgozar, M. Imani, Z. Jahanshiri, M. Shams-Ghahfarokhi, et al., Mater. Sci. Eng., C, 2017, 73, 130-136.

8 S. E. Barnett and S. J. Varley, Ann. R. Coll. Surg. Engl., 1987, 69, 153-155.

9 D. Hoefer, J. K. Schnepf, T. R. Hammer, M. Fischer and C. Marquardt, J. Mater. Sci.: Mater. Med., 2015, 26(4), 1-9.

10 R. Singh and D. Singh, Mater. Med., 2012, 23(11), 2649-2658.

11 I. R. Sweeney, M. Miraftab and G. Collyer, Carbohydr. Polym., 2014, 102(1), 920-927.

12 Y. Qin, Polym. Int., 2008, 57(2), 171-180.

13 Y. Qin, Int. Wound J., 2010, 2(2), 172-176.

14 Y. Qin, J. Appl. Polym. Sci., 2010, 100(3), 2516-2520.

15 M. Rezvanian, M. C. Amin and S. F. Ng, Carbohydr. Polym., 2016, 137(2), 295-304.

16 S. Seetharaman, S. Natesan, R. S. Stowers, C. Mullens, D. G. Baer, L. J. Suggs, et al., Acta Biomater., 2011, 7(7), 2787-2797.

17 R. Raguvaran, B. K. Manuja, M. Chopra, R. Thakur, T. Anand, A. Kalia, et al., Int. J. Biol. Macromol., 2017, 96, 185-191.

18 M. C. Straccia, I. Romano, A. Oliva, G. Santagata and P. Laurienzo, Carbohydr. Polym., 2014, 108(1), 321-330.

19 E. Kang, S. J. Shin, K. H. Lee and S. H. Lee, Lab Chip, 2010, $10(14), 1856$.

20 E. Kang, G. S. Jeong, Y. Y. Choi, K. H. Lee, A. Khademhosseini and S. H. Lee, Nat. Mater., 2011, $10(11), 877$.

21 J. Su, Y. Zheng and H. Wu, Lab Chip, 2009, 9(7), 996-1001.

22 I. Yoo, S. Song, B. Yoon and J. M. Kim, Macromol. Rapid Commun., 2012, 33(15), 1256-1261.

23 J. Cheng, Y. Jun, J. Qin and S. H. Lee, Biomaterials, 2017, 114, 121-143.

24 Y. Jun, E. Kang, S. Chae and S. H. Lee, Lab Chip, 2014, 14(13), 2145-2160.
25 C. H. Mun, J. Y. Hwang and S. H. Lee, Tissue Eng. Regener. Med., 2016, 13(2), 140-148.

26 B. G. Chung, K. H. Lee, A. Khademhosseini and S. H. Lee, Lab Chip, 2012, 12(1), 45-59.

27 X. H. He, W. Wang, K. Deng, R. Xie, X. J. Ju, Z. Liu, et al., RSC Adv., 2014, 5(2), 928-936.

28 E. Kang, Y. Y. Choi, S. K. Chae, J. H. Moon, J. Y. Chang and S. H. Lee, Adv. Mater., 2012, 24(31), 4271-4277.

29 D. Y. Park, C. H. Mun, E. Kang, D. Y. No, J. Ju and S. H. Lee, Biofabrication, 2014, 6(2), 024108-024115.

30 Y. Kitagawa, Y. Naganuma, Y. Yajima, M. Yamada and M. Seki, Biofabrication, 2014, 6(3), 035011-035020.

31 Y. Tian, P. Zhu, X. Tang, C. Zhou, J. Wang, T. Kong, et al., Nat. Commun., 2017, 8(1), 1080.

32 P. Xu, R. Xie, Y. Liu, G. Luo, M. Ding and Q. Liang, Adv. Mater., 2017, 29(34), 1701664.

33 C. H. Yeh, P. W. Lin and Y. C. Lin, Microfluid. Nanofluid., 2010, 8(1), 115-121.

34 Y. Yu, W. Wei, Y. Wang, C. Xu, Y. Guo and J. Qin, Adv. Mater., 2016, 28(31), 6649-6655.

35 S. Wei and Y. L. Hsieh, Carbohydr. Polym., 2014, 102(1), 893900.

36 O. Catanzano, V. D'Esposito, S. Acierno, M. R. Ambrosio, C. C. De, C. Avagliano, et al., Carbohydr. Polym., 2015, 131, 407-414.

37 D. Park, J. Park, H. Jang, J. Cheng, S. H. Kim and S. H. Lee, Biofabrication, 2017, 9(2).

38 S. Partap, I. Rehman, J. Jones and J. Darr, Adv. Mater., 2006, 18(4), 501-504.

39 S. Kirdponpattara, A. Khamkeaw, N. Sanchavanakit, P. Pavasant and M. Phisalaphong, Carbohydr. Polym., 2015, 132, 146-155.

40 Y. Hu and H. Yang, J. Microb. Biochem. Technol., 2015, 07(4), 228-233. 